\title{
Effects of Bothrops moojeni venom on Leishmania amazonensis promastigote forms
}

Castilhos P, Pereira CG, Silva ALN, Napolitano DR, Oliveira F, Souza MA

Erratum to: Effects of Bothrops moojeni venom on Leishmania amazonensis promastigote forms. J Venom Anim Toxins incl Trop Dis. 2011;17(2):150-8.

The article was corrected as requested by its author, Fábio Oliveira. Thus, his affiliation to the National Institute of Science and Technology on Nanobiopharmaceutics (N-BIOFAR) was added to the publication. 